\title{
Novel biocompatible chitosan decorated single-walled carbon nanotubes (SWNTs) for biomedical applications: theoretical and experimental investigations $\dagger$
}

\author{
Sara Piovesan, Paul A. Cox, James R. Smith, Dimitrios G. Fatouros and \\ Marta Roldo*
}

Received 2nd March 2010, Accepted 13th May 2010

DOI: $10.1039 / \mathbf{c 0 0 3 7 6 7 b}$

\begin{abstract}
Molecular mechanics and molecular dynamics simulations have been employed to characterise the interactions between SWNTs and biocompatible amphililic derivatives of chitosan, namely $\mathrm{N}$-butyl- $\mathrm{O}$-sulfate chitosan (NBSC), $\mathrm{N}$-octyl- $\mathrm{O}$-sulfate chitosan (NOSC) and $\mathrm{N}$-palmitoyl- $\mathrm{O}$ sulfate chitosan (NPSC). The computational simulations have shown that the affinity of the polymer for the hydrophobic surface of the nanotubes depends on the length of the chitosan hydrophobic pendant chain. Longer chains have a higher flexibility and therefore a better ability to wrap around the nanotubes. To underpin the theoretical calculations, experimental studies revealed that NPSC exhibits highest affinity for SWNTs with up to $66.9 \pm 19.7 \%$ SWNTs stably suspended in an aqueous environment; this affinity was confirmed by the calculated binding energy of five polymer chains with a SWNT that was found to be $-300.93 \mathrm{kcal} \mathrm{mol}^{-1}$, the highest amongst the three polymers studied. Furthermore, the high value of cell viability after incubation with NPSC indicates that this is a good candidate for the preparation of biocompatible SWNTs dipersions that could be used in biomedical and pharmaceutical applications.
\end{abstract}

\section{Introduction}

Carbon nanotubes (CNTs) are allotropes of carbon, composed of carbon atoms arranged into a series of graphene sheets, "rolled" into a cylindrical structure. They belong to the family of fullerenes, the third allotrope of carbon after graphite and diamond. ${ }^{1-3}$ CNTs are highly versatile due to their physicochemical features. They possess ordered structures with a high aspect ratio and ultra-light weight. They have high mechanical and tensile strengths, and high electrical and thermal conductivity. They exhibit both semi-metallic and metallic behaviour and have large surface areas. ${ }^{3}$ Furthermore, they possess outstanding chemical and thermal stability. ${ }^{4}$

The interaction between cells and CNTs, and hence their internalisation into cells, needs to be clarified in order to ascertain their future potential as drug delivery systems. ${ }^{5}$ Nevertheless, numerous studies have been carried out using biocompatible CNTs whereby CNTs have undergone covalent or non-covalent functionalisation rendering them soluble in aqueous media and hence biologically compatible. ${ }^{6}$ In noncovalently functionalised CNT dispersions, the stability of the dispersion depends on the efficiency of the physical wrapping of molecular units around the CNTs. This "physical wrapping" involves forces that are relatively weaker than those involved in covalent functionalisation and hence the

School of Pharmacy and Biomedical Sciences,

University of Portsmouth, St. Michael's Building, White Swan Road, Portsmouth PO1 2DT, UK. E-mail: marta.roldo@port.ac.uk;

Fax: + 44 (023) 9284 3565; Tel: + 44 (023) 92843586

$\dagger$ Electronic supplementary information (ESI) available: Method for cytotoxicity assay; NMR, ATR, XRD data; further AFM topography images. See DOI: 10.1039/c003767b latter is expected to produce the most stable dispersion. However, covalent functionalisation alters the electronic structure of CNTs and hence potentially also affects their physical properties. ${ }^{2}$ Therefore, non-covalent chemical modification of CNTs is particularly attractive as it offers the facility of associating functional groups with the CNT surface without modification of the $\pi$ system of the graphene lattice and hence not modifying their electrical or physical properties. $^{7}$

Molecular mechanics (MM) and molecular dynamics (MD) simulations have become increasingly important in the investigation of the interactions occuring at the interface of CNT-polymer systems due to the difficulties in exploring their properties by experimental methods. In a recent study, MM and MD simulations were combined with experimental studies to shed light on the interactions taking place in the formation of polymer assisted carbon nanotubes dispersions. ${ }^{8}$ The interfacial bonding of SWNTs $(10,10)$-epoxy composites was studied using a combination of experimental and computational methods. The mechanical behaviour of the system was investigated by MD analysis while the interfacial bonding was evaluated from MM energies. The results obtained showed that the interaction energy is due to both van der Waals and electrostatic interactions between the nanotube and the epoxy resin. ${ }^{9}$ Yang et al. used MD simulations to study the intramolecular interaction energy between SWNTs and different polymers, such as polystyrene, poly(phenyl-acetylene), and others. ${ }^{10}$ MD simulations were first carried out on a SWNT interacting with an individual polymer chain; then a series of MD simulations were run to investigate the wrapping process of the polymers around a SWNT. In this study, it was found that the specific monomer structure plays a very important role 
in determining the interaction between the carbonaceous surface and the polymer. To investigate the relative arrangement of polymer aromatic rings with respect to the carbon nanotube suface, dihedral angles between the surface and the plane of aromatic rings were calculated. It was found that SWNTpolymer interactions are strongest for conjugated polymers with aromatic rings on the polymer backbone as these rings are able to align parallel to the nanotube surface and thereby provide strong interfacial adhesion. ${ }^{10} \mathrm{MM}$ and MD simulations were also used to explore the interfacial interaction occurring during the noncovalent association of SWNTs with poly(ethylene) (PE). ${ }^{11}$ The negative interaction energy obtained for this system, indicated that PE molecules move towards the surface of the nanotube due to attractive van der Waals interactions. ${ }^{11}$ All of these studies have demonstrated that the presence of hydrophobic domains in the polymeric structure play a paramount role in the interaction with CNTs.

In a recent study, we employed MM and MD simulations and experimental studies to investigate the interaction of SWNTs with the biocompatible chitosan derivative, $N$-octyl- $O$-sulfate chitosan (NOSC). ${ }^{12}$ The computational data allowed the calculation of the interaction energy between the SWNT surface and the polymer and also to determine the number and conformation of the polymeric chains that interact with the carbonaceous surface. Furthermore, the in silico model correctly predicted the diameter of the wrapped SWNTs which was then confirmed by atomic force microscopy. ${ }^{12}$ As a follow up study, herein we present the effect of the length of the chitosan derivative side chain on the affinity of the polymers for SWNTs. The computational simulations are compared with experimental loading and stability studies. Butyl and palmitoyl analogues of NOSC were prepared and their biocompatibility was also tested.

\section{Materials and methods}

\section{Materials}

Low viscosity chitosan, chlorsulfonic acid, pyrene and sodium hydroxide $(\mathrm{NaOH})$ were purchased from Fluka Biochemika, UK. Methanol (MeOH), dimethylformamide (DMF), acetone, glacial acetic acid and sodium chloride $(\mathrm{NaCl})$ were provided by Fisher Scientific, Loughborough, UK. Octaldehyde was from Sigma-Aldrich, Poole, UK, whilst butyraldehyde, palmitoyl chloride and sodium borohydrate $\left(\mathrm{NaBH}_{4}\right)$ were purchased from Acros Organics, Poole, UK. HipCO SWNTs were obtained from Carbon Nanotechnology Inc., Houston, TX, USA (CNI Grade/Lot\#: P0332). All solutions were prepared with Millipore water (conductivity $<0.5 \mu \mathrm{S} \mathrm{cm}^{-1}$ ).

\section{Synthesis of chitosan derivatives}

Synthesis of $\mathrm{N}$-octyl chitosan and $\mathrm{N}$-octyl- $\mathrm{O}$-sulfate chitosan was performed as previously described. ${ }^{12,13}$ Characterisation data have also been reported. ${ }^{13}$

\section{Synthesis of $N$-butyl chitosan (NBC)}

Chitosan (1.0 g) was suspended in methanol $(50 \mathrm{~mL})$ and butyraldehyde $(1.2 \mathrm{~g})$ was added to the suspension while stirring; the suspension obtained was stirred at room temperature for $24 \mathrm{~h}$. An aqueous solution of $\mathrm{NaBH}_{4}(0.5 \mathrm{~g}$ in $5 \mathrm{~mL}$ ) was slowly added to the reaction mixture and the resulting mixture was stirred at room temperature for a further $24 \mathrm{~h}$. The reaction was stopped by neutralisation with $2 \mathrm{M}$ $\mathrm{HCl}$. The product was filtered and repeatedly washed with methanol and water and finally dried under vacuum at $60{ }^{\circ} \mathrm{C}$ to constant weight $(1.16 \pm 0.14 \mathrm{~g}, n=4)$.

\section{Synthesis of $\mathrm{N}$-butyl- $\mathrm{O}$-sulfate chitosan (NBSC)}

NBC (1.0 g) was suspended in DMF (40 mL). Chlorsulfonic acid $(20 \mathrm{~mL})$ was added dropwise to DMF $(40 \mathrm{~mL})$ and the mixture stirred for $1 \mathrm{~h}$ at $0{ }^{\circ} \mathrm{C}$. The $N$-alkyl-chitosan suspension was then added to the above solution. The mixture was reacted at $40{ }^{\circ} \mathrm{C}$ for $24 \mathrm{~h}$ and the reaction stopped by neutralisation with $\mathrm{NaOH}(20 \% \mathrm{w} / \mathrm{v})$; the obtained precipitate was filtered off and the filtrate was dialysed against distilled water for 3 days and then freeze dried $(0.25 \pm 0.10 \mathrm{~g}, n=3)$.

\section{Synthesis of $\mathrm{N}$-palmitoyl- $O$-sulfate chitosan (NPSC)}

Chitosan ( $2 \mathrm{~g})$ was dissolved in acetic acid $(0.12 \mathrm{M}, 240 \mathrm{~mL})$ and the $\mathrm{pH}$ of the solution was adjusted to 7.2 by slow addition of $\mathrm{NaOH}(0.1 \mathrm{M})$. Palmitoyl chloride $(4.4 \mathrm{~g})$ was then added and the solution stirred overnight. At the end of the reaction, the $\mathrm{pH}$ of the solution was adjusted to 7.0 and the polymer was precipitated in acetone. The mixture was sonicated for $5 \mathrm{~min}$ to eliminate air bubbles from the precipitate and then centrifuged at $2000 \mathrm{rpm}$ for $5 \mathrm{~min}$. The precipitate was finally washed with hot methanol $(\times 2)$ and centrifuged at $2000 \mathrm{rpm}$ for $5 \mathrm{~min}$. The polymer collected was suspended in DMF $(80 \mathrm{~mL})$ and then added to a mixture of chlorsulfonic acid $(30 \mathrm{~mL})$ and DMF $(80 \mathrm{~mL})$ previously prepared in an ice bath. The reaction mixture was stirred at $40{ }^{\circ} \mathrm{C}$ for $24 \mathrm{~h}$. The reaction was stopped by neutralisation with $\mathrm{NaOH}(20 \% \mathrm{w} / \mathrm{v})$ and filtration through a micro glass fibre filter followed by washing with water $(\times 2)$ and the resulting polymer solution was further purified by dialysis against deionised water for 3 days. After freeze drying, an off-white material was obtained $(0.21 \pm 0.14 \mathrm{~g}, n=3)$.

\section{Characterisation of chitosan derivatives}

Chitosan derivatives were characterised by the following methods. ${ }^{1} \mathrm{H}$ NMR spectra were obtained on a JEOL $400 \mathrm{MHz}$ spectrometer operating at $400 \mathrm{MHz}$. The samples were dissolved in $\mathrm{D}_{2} \mathrm{O}$ and TMS was used as a standard. ATR spectra were recorded on a Tensor 27 FTIR spectrophotometer. X-ray diffraction studies were conducted on a Philips X-ray diffractometer. Elemental analysis was carried out with a Carlo-Erba CHNS Elemental Analyzer EA1108. The degree of substitution, i.e. alkylation or acylation, was calculated using the method reported by Choi et al. ${ }^{14}$ with a modified formula:

$$
\text { DSub }=[(\% \mathrm{C} / 12.001) /(\% \mathrm{~N} / 14.007)] / \mathrm{X}
$$

where $\mathrm{X}$ is 10 for the butyl derivative, 14 for the octyl derivative and 22 for the pamitoyl derivative. The degree of sulfation (DS) was calculated using the formula: ${ }^{15}$

$$
\mathrm{DS}=(\% \mathrm{~S} / 32.06) /(\% \mathrm{~N} / 14.007)
$$




\section{Critical micelle concentration (cmc) determination}

The critical micelle concentration $(\mathrm{cmc})$ of chitosan derivatives was determined using pyrene as a hydrophobic probe. ${ }^{12,16}$

\section{Biocompatibility studies}

The three polymers synthesised were tested to determine their biocompatibility in terms of blood compatibility and Caco-2 cell viability. The anticoagulant activity of chitosan derivatives was determined according to a method previously published using the Amax ${ }^{\circledR}$ Heparin assay kit (Trinity Biologicals, Dublin, IR). ${ }^{12}$ The cytotoxycity was investigated performing an MTT assay (see ESI for method). ${ }^{17}$

\section{Purification of carbon nanotubes and preparation of aqueous suspensions}

Microwave assisted purification of SWNTs was carried out as described in the literature with slight modification. ${ }^{18}$ Carbon nanotubes $(20 \mathrm{mg})$ were placed into a $100 \mathrm{~mL}$ conical flask. The nanotubes were placed into a conventional microwave at $90 \mathrm{~W}$ for $5 \mathrm{~s}$; the nanotubes were then removed from the microwave, shaken and stirred with a spatula, and placed back into the microwave for another $5 \mathrm{~s}$ of treatment; this process was repeated sixty times. The SWNTs were then suspended in concentrated $\mathrm{HCl}(10 \mathrm{~mL})$, the mixture obtained was centrifuged at $4000 \mathrm{rpm}$ for $5 \mathrm{~min}$. After discarding the supernatant, the SWNTs were further washed with water $(10 \mathrm{~mL})$, methanol $(10 \mathrm{~mL})$, and diethyl ether $(10 \mathrm{~mL})$. The remaining solid was dried using compressed air. A total amount of $10.8 \mathrm{mg}$ of purified SWNTs was obtained.

Stable dispersions of purified SWNTs were obtained by mixing pristine material (final concentration $0.1 \mathrm{mg} / \mathrm{mL}$ ) and NOSC, NBSC or NPSC (10 mL, $0.1,0.3$ and $0.5 \mathrm{mg} / \mathrm{mL})$ and subjecting the mixture to sonication in an ultrasonic bath for $2 \mathrm{~h}$. The dispersions were then centrifuged at $6000 \mathrm{rpm}$ for 3 min to eliminate any unstable bundles still present in suspension.

\section{Characterisation of SWNTs suspensions and AFM studies}

The apparent absorption coefficient of SWNTs dispersed in NBSC, NOSC and NPSC solutions was determined as previously described. ${ }^{12,19}$ The values obtained were used to calculate the concentration of the SWNTs present in the suspensions. Samples for atomic force microscopy (AFM) studies were prepared by placing a drop $(10 \mu \mathrm{L})$ of the polymer-SWNT suspension on freshly cleaved muscovite mica (Agar Scientific, Stansted, Essex, UK), mounted on a nickel disc (dia. $\left.1 \mathrm{~cm}^{2}\right)$ with double-sided adhesive tape, left for $2 \mathrm{~min}$ and excess liquid removed with a gentle stream of $\mathrm{N}_{2}$. AFM studies were carried out using a MultiMode/NanoScope IV Scanning Probe Microscope (Digital Instruments, Santa Barbara, CA, USA) in air under ambient conditions $\left(T=23{ }^{\circ} \mathrm{C}\right.$, $R H=21 \%$ ) using the $J$-scanner $(\max . x y=200 \mu \mathrm{m})$. Scanning was performed in Tapping Mode using Si cantilevers with integrated tips $(t=3.5-4.5 \mu \mathrm{m}, l=115-135 \mu \mathrm{m}$, $w=30-40 \mu \mathrm{m}, \nu_{0}=200-400 \mathrm{kHz}, k=20-80 \mathrm{~N} \mathrm{~m}^{-1}$, $R<10$ nm; Model: RTESP, Veeco Instruments, France) and an RMS amplitude of $0.8 \mathrm{~V}$ was used. Images were subsequently processed using NanoScope software
(V 7.10, Digital Instruments, Santa Barbara, CA, USA). Diameters of coated and uncoated SWNTs were calculated from height values that are less susceptible to tip-sample artefact errors than lateral diameter values. ${ }^{12}$

\section{Computational methods}

A combination of molecular dynamics and energy minimisation methods were used to investigate the mode of binding of the NBSC, NOSC and NPSC polymer chains to the surface of the nanotubes. Simulations were performed using the CVFF forcefield $^{20}$ as implemented in the program Materials Studio 4.1 (Accelrys Software Inc, San Diego, CA, USA). An $(8,8)$ single walled nanotube was constructed with an overall length of $150 \AA$ and terminated with hydrogen atoms. Polymer chains consisting of 70 repeat units were initially energy minimised in the absence of the nanotube. Polymer molecules were then docked within $5 \AA$ from the surface of the nanotube and a combination of molecular dynamics and molecular mechanics were used to deterimine the lowest energies for the polymers relative to the nanotube. This was carried out by performing molecular dynamics at high temperature $(750 \mathrm{~K})$ for several thousand time-steps (typically $200000 \mathrm{steps}, \Delta t=1 \times 10^{-15} \mathrm{~s}$ ) followed by energy minimisation using a conjugate gradient algorithm until a convergence force of $0.001 \mathrm{kcal} \mathrm{mol}^{-1} \AA$ was obtained. This sequence was repeated until no further decrease in the energy was obtained. Calculations were performed first using one polymer chain and then five chains. Five chains were used as an earlier investigation suggested that this gave the best binding energy for the NOSC polymer. ${ }^{12}$

\section{Results and discussion}

\section{Synthesis of NBSC}

Chitosan was modified by alkylation of the free amino group in position $\mathrm{C} 2$ with butyraldehyde. The product was successfully obtained in the form of an off-white powder. The degree of modification obtained was 0.82 , higher than that obtained for NOC (0.77). ${ }^{13}$ The higher modification degree can be explained by the lower steric hindrance of the butyl chain that leads to a higher reactivity of the aldehyde towards the polysaccharide (Table 1).

The second step of the reaction involved the sulfation of NBC in chlorsulfonic acid and DMF; the sulfation degree obtained was only 0.47 , this could be due to the limited solubility of NBC in DMF. The low degree of sulfation can

Table 1 Physicochemical characterisation of the polymers: elemental analysis data and calculated degree of substitution (DSub) in terms of alkylation or acylation and sulfation (DS) of chitosan derivatives; critical micelle concentration (CMC) is given in $\mathrm{mg} / \mathrm{mL}$

\begin{tabular}{lcccccl}
\hline Polymer & $\% \mathrm{C}$ & $\% \mathrm{~N}$ & $\% \mathrm{~S}$ & $\mathrm{DSub}$ & $\mathrm{DS}$ & $\mathrm{CMC}$ \\
\hline NBC & 47.10 & 6.70 & - & 0.82 & - & - \\
NBSC & 38.89 & 5.19 & 5.24 & 0.87 & 0.47 & $0.023 \pm 0.007 * * *$ \\
NOC & 53.96 & 5.67 & - & 0.79 & - & - \\
NOSC & 18.93 & 2.78 & 7.54 & 0.57 & 1.19 & $0.095 \pm 0.008$ \\
NPSC & 20.37 & 4.86 & 6.97 & 0.22 & 0.63 & $0.030 \pm 0.017 * *$ \\
One-way & Anova: $p=$ & 0.0005. & Tukey & Kramer: **p & $<0.01$; \\
$* * * p<0.001$ compared to NOSC.
\end{tabular}


also explain the low yield of the reaction as limited sulfation leads to the formation of a very poorly soluble polymer that is then lost during the purification process. The low degree of sulfation is also reflected in that the degree of alkylation had not decreased as was previously observed in the case of the NOSC polymer. ${ }^{13}$ Successful alkylation with the butyl chain was demonstrated by ATR; the disappearance of the peaks assigned to the primary amino group of chitosan $\left(-\mathrm{NH}_{2}\right.$ bending vibrations at 1650 and $1590 \mathrm{~cm}^{-1}$ ) was observed (Fig. A area (iii), see ESI), $\dagger$ whilst new peaks attributed to the alkyl chain were identified at 2961 and $2874 \mathrm{~cm}^{-1}$ (Fig. A area (ii), see ESI). ${ }^{21-23} \dagger$ The ATR spectra also revealed that the introduction of the butyl group has a less important effect on the intermolecular hydrogen bonding of the polysaccharide as compared to that of the octyl chain (Fig. A area (i), see ESI). ${ }^{13} \uparrow$ This is demonstrated by the reduction in intensity of the two peaks in the region of $3100-3300 \mathrm{~cm}^{-1}$, assigned to the $\mathrm{N}-\mathrm{H}$ and $\mathrm{O}-\mathrm{H}$ stretching restricted by the hydrogen bonding; ${ }^{24}$ this is less accentuated for NBC compared to NOC, confirming that the lower solubility of the derivative could be the cause of the lower degree of sulfation obtained. ATR analysis also revealed that $O$-sulfation occurred mainly at the hydroxyl group in position $\mathrm{C} 6$ as the peak attributed to the combination of $\mathrm{O}-\mathrm{H}$ bending and $\mathrm{C}-\mathrm{O}$ stretching of the primary alcohol $\left(1150 \mathrm{~cm}^{-1}\right)$ disappeared and new peaks assigned to $\mathrm{O}=\mathrm{S}=\mathrm{O}$ and C-O-S appeared at 1249, 1211, 995, $805 \mathrm{~cm}^{-1}$ (Fig. A area (iv), see ESI). ${ }^{15,25-30} \dagger$ Furthermore, modification was also visible on the ${ }^{1} \mathrm{H}-\mathrm{NMR}$ spectrum (Table $\mathrm{A}$, see ESI) $\dagger$ in which new peaks at $0.9-1.1 \mathrm{ppm}\left(-\mathrm{NH}-\mathrm{CH}_{2}-\left(\mathrm{CH}_{2}\right)_{2}-\mathrm{CH}_{3}\right)$, 1.3-1.8 ppm (-NH- $\left.\mathrm{CH}_{2}-\left(\mathrm{CH}_{2}\right)_{2}-\mathrm{CH}_{3}\right)$ and 3.7-3.8 ppm $\left(-\mathrm{NH}-\mathrm{CH}_{2}-\left(\mathrm{CH}_{2}\right)_{2}-\mathrm{CH}_{3}\right)$ were observed. The ${ }^{1} \mathrm{H}-\mathrm{NMR}$ spectrum also confirmed the successful sulfation in position C6 (two broad peaks at 3.6-3.9 and 3.9-4.1 ppm). ${ }^{31}$ The NBC XRD pattern showed the peak at $2 \theta=20^{\circ}$, characteristic of the chitosan form II crystal, while the peak at $2 \theta=11^{\circ}$ disappeared and a new peak at $2 \theta=5^{\circ}$ was observed, in agreement with data obtained for NOC (Fig. B, see ESI). ${ }^{13,32} \dagger$ Loss of crystallinity was observed for the NBSC derivative, that showed only weak diffraction peaks.

\section{Synthesis of NPSC}

Acylation of low molecular weight chitosan was obtained by reaction of the free amino group in position $\mathrm{C} 2$ with palmitoyl chloride. The product of the first step of the reaction was not isolated but rather used directly for the sulfation step. $N$-palmitoyl- $O$-sulfate chitosan was successfully obtained in the form of a white material with a degree of acylation of only 0.22 , much lower than that obtained for the butyl and octyl derivatives; probably because of the much higher steric hindrance of the longer carbon chain. The degree of sulfation was of 0.63 . Successful acylation with the palmitoyl chain has been shown by ATR (Fig. Ad, see ESI); $\dagger$ modifications similar to those described above in the case of the butyl derivative were evident in the spectra. Furthermore, the absence of a peak around $1700-1800 \mathrm{~cm}^{-1}$, assigned to the presence of esters, confirmed that no $O$-acylation had occurred. ${ }^{33}$ Formation of the secondary amide is not only confirmed by the increased intensity of the peak at $1655 \mathrm{~cm}^{-1}$ (amide I band, $\mathrm{C}=\mathrm{O}$ stretching vibration, (Fig. Ad area (iii), ESI), $\dagger$ but also by the intensification of the bands at $3100-3300 \mathrm{~cm}^{-1}$ (Fig. Ad area (i), see ESI) $\dagger$ assigned to the $\mathrm{N}-\mathrm{H}$ and $\mathrm{O}-\mathrm{H}$ stretching restricted by the hydrogen bonding; peaks that did disappear when alkylation was carried out instead of acylation. ${ }^{24}$ Moreover, modification was shown also on the ${ }^{1} \mathrm{H}-\mathrm{NMR}$ spectrum in which new peaks between 1.1 and $2.5 \mathrm{ppm}$ (Table A, see ESI), $\dagger$ assigned to the $\mathrm{CH}_{2}$ protons of the acyl chain, were observed. Overlapping of peaks did not allow the identification of the signals assigned to the protons of the sulfated C6. ${ }^{31}$ The XRD pattern of NPSC (Fig. Bd, see ESI) $\dagger$ showed complete loss of crystallinity.

\section{Critical micelle concentration (cmc)}

The cmc of both NBSC and NPSC has been found to be significantly lower than that of NOSC (Table 1). ${ }^{13}$ This can be explained by the lower degree of sulfation obtained for these two polymers. A smaller number of sulfate groups along the polymer would favour hydrophobic interactions amongst the pendant carbon chains as there is reduced stretching of the polymeric backbone due to repulsion between sulfate groups compared to the NOSC polymer. Therefore, in terms of micellar stability, a lower degree of sulfation seems to be more favourable.

\section{Polymers biocompatibility studies}

The effect of the hydrophobic chain length on the anticoagulant activity of these polymers was also studied (Fig. 1). No significant anticoagulant activity was observed for NBSC or NPSC in the concentration range 0 to $5 \mathrm{mg} / \mathrm{mL}$. This is due to the low degree of sulfation obtained for these polymers.

With degree of sulfation of 0.47 and 0.63 , respectively, neither of the polymers presents a high affinity for antithrombin III. ${ }^{31}$ The two non active polymers were further tested for their cytotoxicity when incubated with Caco-2 cells (Fig. 2). All polymers showed a significantly higher cell viability when compared with $\operatorname{SDS}(p<0.0001$, Tukey Kramer). NBSC reduced only marginally the cell viability with no statistical difference between concentrations $(p>0.05$, Tukey Kramer). Whilst NPSC showed a concentration dependent effect on cell viability, with significant reductions observed

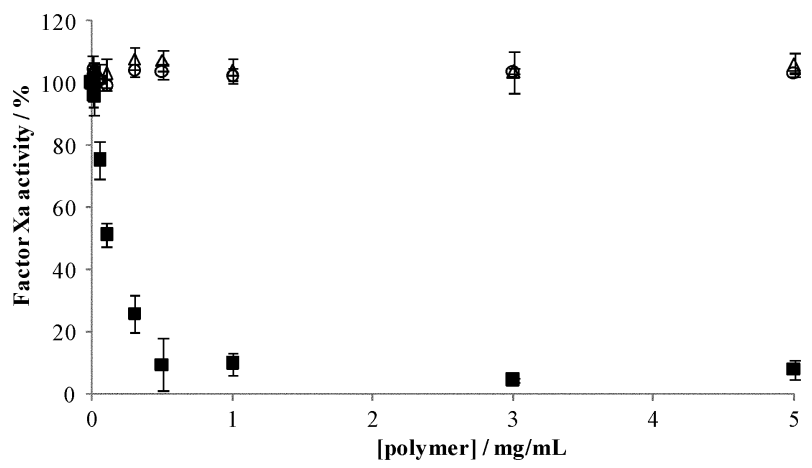

Fig. 1 Anticoagulant activity of $\mathrm{N}$-butyl- $O$-sulfate chitosan (empty circle), $\mathrm{N}$-octyl- $\mathrm{O}$-sulfate chitosan (black square) and $\mathrm{N}$-palmitoyl- $\mathrm{O}$ sulfate chitosan (empty triangle). Data are given as mean $\pm \mathrm{SD}$ $(n=3)$. 


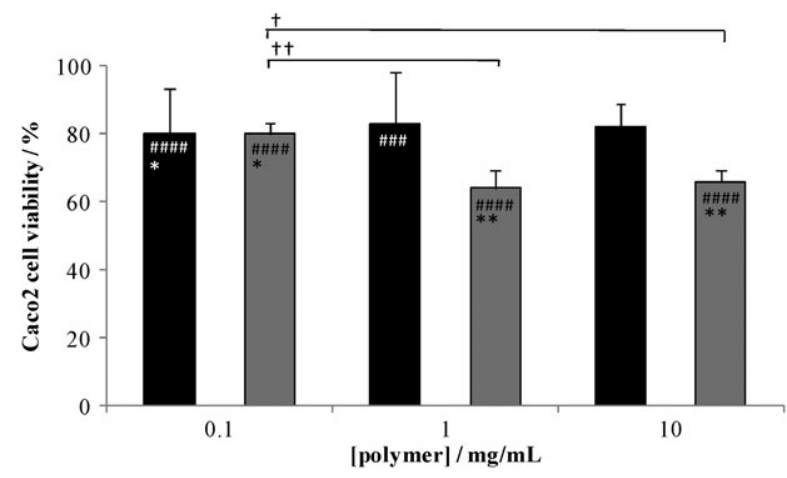

Fig. 2 Caco-2 cell viability after incubation with chitosan derivatives: NBSC (black) and NPSC (grey). Results are reported as mean \pm SD $(n=3)$. Two tailed $t$-test: ${ }^{*} p<0.05,{ }^{* * *} p<0.01$ compared to medium; \#\#\# $p<0.001$, \#\#\#\# $p<0.0001$ compared to SDS. Anova, Tukey Kramer post-hoc test: $\dagger p<0.05, \dagger \dagger p<0.01$.

only for concentrations above $1 \mathrm{mg} / \mathrm{mL}$. NOSC biocomaptibility was demostrated in tests with Artemia, reported elsewere. ${ }^{13}$

\section{Characterisation of SWNTs suspensions in aqueous solutions of chitosan derivatives}

Chitosan derivatives are able to thermodynamically stabilise SWNTs by disrupting the intertubular attractions and reducing hydrophobicity of the carbon surface in contact with water. ${ }^{34}$

In particular, the SWNT's surface will come into contact with the hydrophobic chains present on the amphiphilic derivatives of chitosan and therefore the length of these chains is predicted to have a major effect on the stabilisation of the carbon nanotubes in an aqueous environment (Fig. 3). NBSC was found to have a similar behaviour to NOSC, with no evident advantage. The maximum loading obtained with this polymer was of only $27.7 \pm 17.2 \%$. On the other hand NPSC, presenting a longer side chain, achieved a maximum loading of $66.9 \pm 19.7 \%$, significantly higher than the values obtained with NOSC $(p<0.05)$. NPSC-SWNTs suspensions have also been shown to be stable for prolonged time at room temperature with non-statistically significant (Anova, $p>0.05$ ) decrease in number of suspended carbon nanotubes over a period of 30 days (Fig. 4).

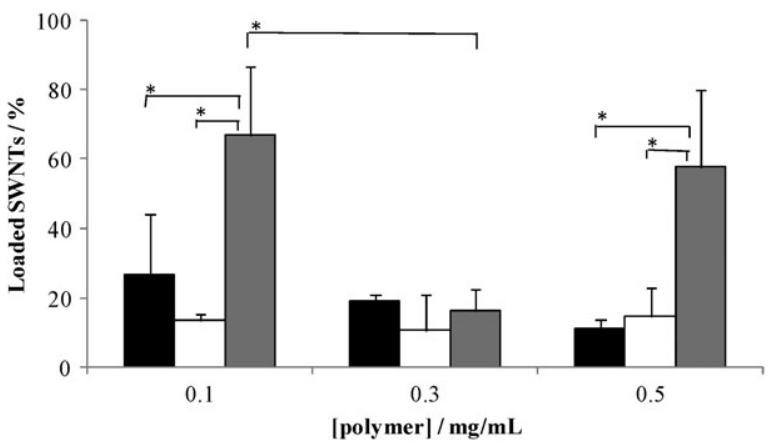

Fig. 3 SWNTs loading capacity of chitosan derivatives: NBSC (black), NOSC (white) and NPSC (grey). Results are reported as mean $\pm \mathrm{SD}(n=3)$. One-way Anova, Tukey-Kramer post-hoc test: $* p<0.05$.

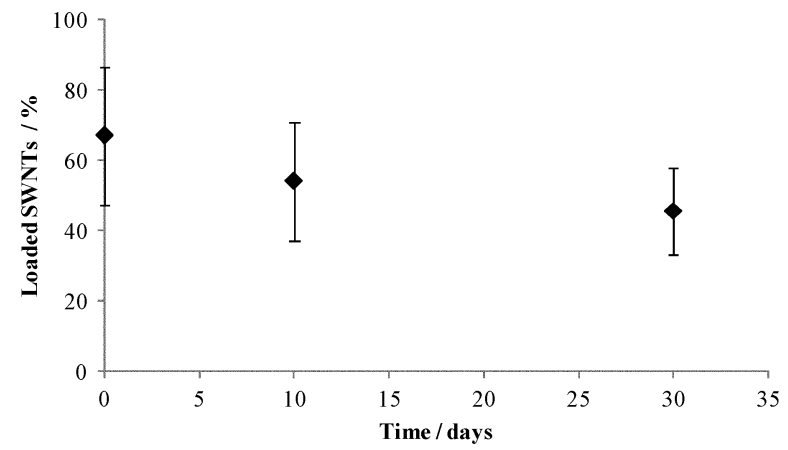

Fig. 4 Stability studies of SWNTs/NPSC suspensions prepared with polymer solutions of $0.1 \mathrm{mg} / \mathrm{mL}$. Results are reported as mean $\pm \mathrm{SD}$ $(n=3)$. One-way Anova, $p>0.05$.

\section{AFM studies}

Significant variations were observed among the different polymers used for the stabilisation of the carbonaceous material as discerned from AFM topography images (Fig. 5).

As in previous studies, a few nanotubes had regions which were uncoated, with typical height measurements of $1.2 \pm 0.7 \mathrm{~nm}$ (Table 2).

Small numbers of nanotubes were observed when the NBSC polymer was used to assist their dispersion (Fig. 5, left panel). Their length was $c a .1 \mu \mathrm{m}$ and their height was $2.9 \pm 1.1 \mathrm{~nm}$ $(n=30)$, significantly higher than the uncoated nanotubes ( $p<0.001$, Tukey Kramer). In contrast, the NOSC polymer can assist in the assembly of SWNT structures, with the formation of Y-junctions (Fig. 5, middle). Such structures have been reported previously with a series of peptides and were partly attributed to a peptide-assisted end-to-end SWNT assembly. ${ }^{35}$ Moreover, the number of the individual nanotubes was increased when the NOSC derivative was used for the stabilisation of the nanotubes. The height of the NOSC-coated regions on the nanotubes was $3.2 \pm 1.0 \mathrm{~nm}(n=40)$, only marginally larger than that of the NBSC-coated material ( $p<0.05$, Tukey Kramer). Similarity in behaviour between NBSC and NOSC was equally observed in the loading studies (Fig. 3). The most pronounced effect to the dispersability of the nanotubes was noticed in the presence of NPSC, as illustrated in Fig. 5, right panel. Large numbers of short nanotubes were produced in the presence of this polymer corroborating the high loading values obtained with this polymer (Fig. 3). The height of these coated nanotubes was $5.6 \pm 1.6 \mathrm{~nm}(n=40)$, statistically higher than the diameters obtained for the other polymers ( $p<0.001$, Tukey Kramer). The height (diameter) distributions of these hybrids are illustrated in Fig. 6.

\section{Computational modelling}

The energy minimised structures of the three polymer molecules showed that they all adopt a helical backbone, with the alkyl chains radiating outwards (Fig. 7).

The helical conformation of the chitosan backbone has been shown to be caused by the formation of intramolecular hydrogen bonding between the hydrogen atom of the hydroxyl group in position $\mathrm{C} 3$ and the oxygen atom of the glucosamine ring of the adjacent monomer (Scheme 1). ${ }^{36}$ 

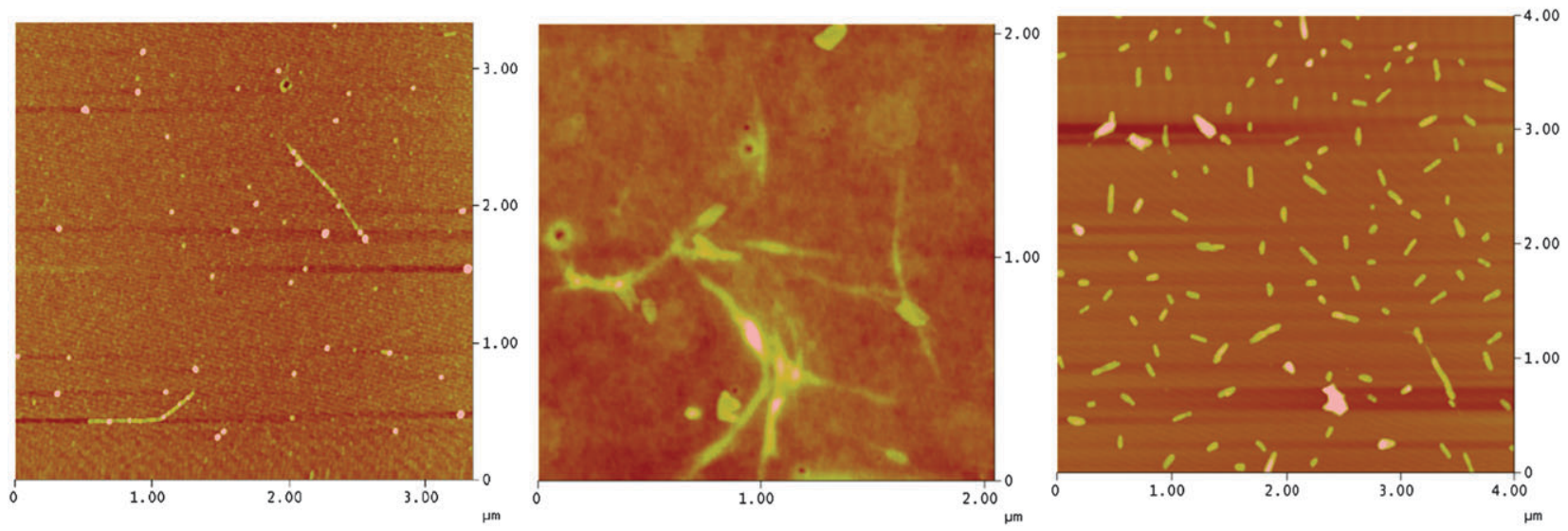

Fig. 5 AFM topography images of SWNTs coated with NBSC, NOSC and NPSC (from left to right).

Table 2 AFM determined and calculated (molecular modelling) diameters for SWNTs coated with the three polymers. Experimental data are given as mean $\pm \mathrm{SD}(n=30$ for NBSC and 40 for NOSC and NPSC)

\begin{tabular}{lll}
\hline Polymer & $\begin{array}{l}\text { Measured } \\
\text { diameter } / \mathrm{nm}\end{array}$ & $\begin{array}{l}\text { Calculated } \\
\text { diameter } / \mathrm{nm}\end{array}$ \\
\hline No polymer & $1.2 \pm 0.7$ & - \\
NBSC & $2.9 \pm 1.1$ & 3.9 \\
NOSC & $3.2 \pm 1.0$ & 4.7 \\
NPSC & $5.6 \pm 1.6$ & 5.3 \\
\hline
\end{tabular}

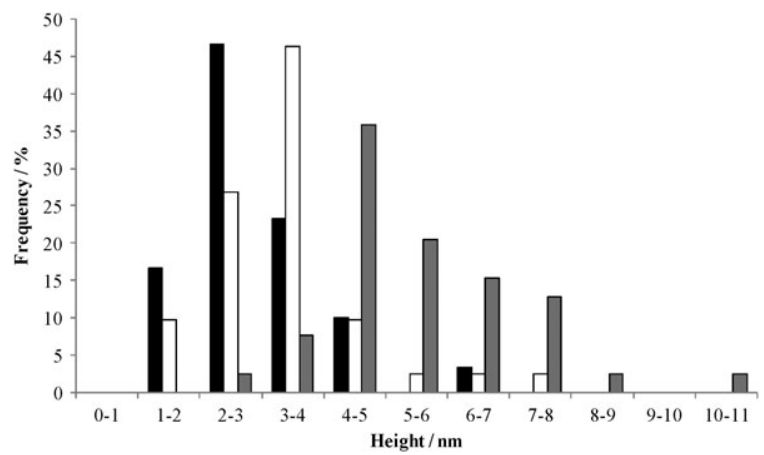

Fig. 6 Height distributions, determined from AFM measurements, of SWNTs coated with NBSC (black bar; $n=30$ ), NOSC (grey bar; $n=40$ ) and NPSC (white bar; $n=40$ ).

Therefore, the modifications induced by alkylation/acylation and sulfation of the monomers, which involve positions $\mathrm{C} 2$ and C6 (Scheme 1), do not seem to affect the formation of the intramolecular interactions. The optimised binding energies for the complexes between the three polymers and SWNTs are shown in Table 3 . The results show that interaction between each of the polymers with the nanotube is energetically favourable. With a single polymer chain optimised on the surface of the nanotube, it can be seen that the binding energy is considerably bigger for the NPSC polymer than the other two. NBSC and NOSC present very similar binding energies which were experimentally reflected in the similar loading capacity (Fig. 3). Optimum energies are obtained when the polymer molecules are aligned along the length of the nanotube, this maximises the van der Waals interaction between the polymeric chain and the carbonaceous surface. When rotated

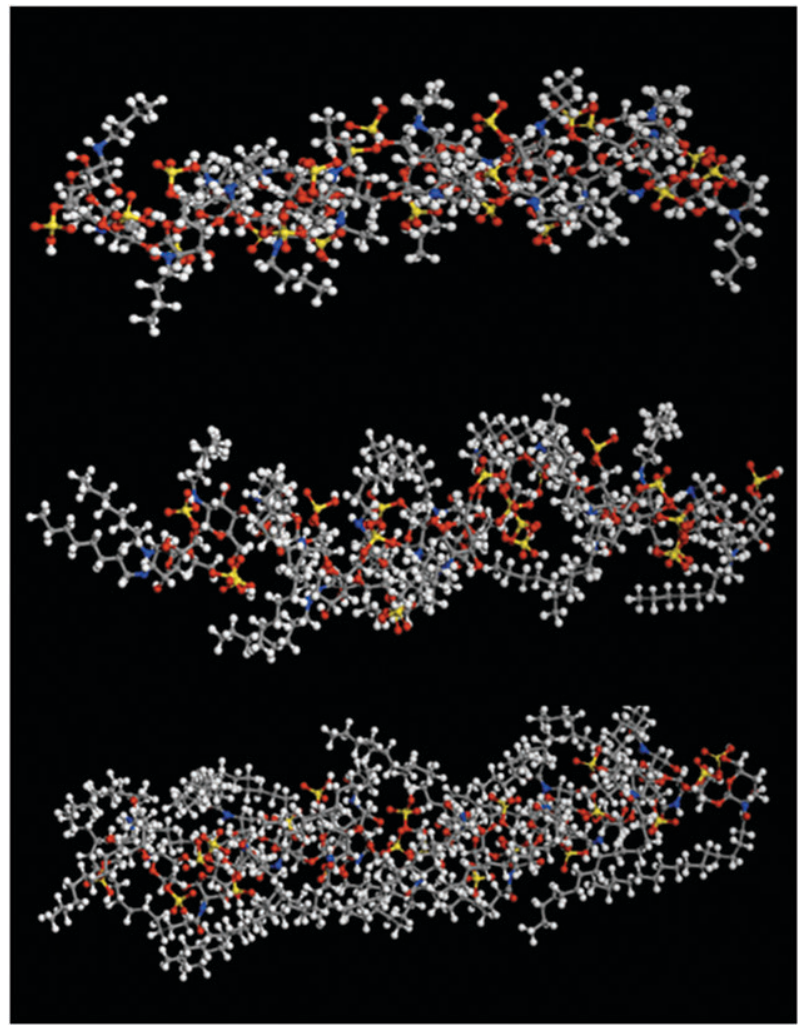

Fig. 7 Polymeric chain conformation of NBSC (top), NOSC (middle) and NPSC (bottom).

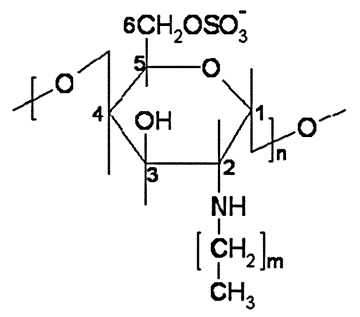

(I)

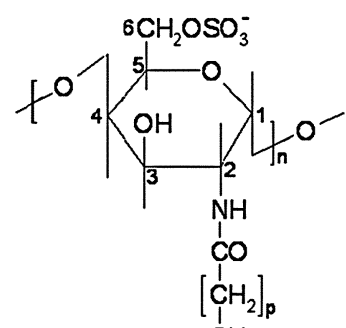

(II)
Scheme 1 Structure of NBSC (I, $m=3), \operatorname{NOSC}(\mathrm{I}, m=7)$ and $\operatorname{NPSC}(p=14)$. 
Table 3 Binding energies obtained for the three polymer molecules with 1 and 5 chains

\begin{tabular}{lll}
\hline Polymer & $\begin{array}{l}\text { Number of polymer } \\
\text { chains bound to SWNT }\end{array}$ & $\begin{array}{l}\text { Binding Energy } \\
\text { per chain } / \mathrm{kcal} \mathrm{mol}^{-1}\end{array}$ \\
\hline NBSC & 1 & -123.55 \\
NOSC & 1 & -123.06 \\
NPSC & 1 & -272.21 \\
NBSC & 5 & -208.08 \\
NOSC & 5 & -286.47 \\
NPSC & 5 & -300.93 \\
\hline
\end{tabular}

by $90^{\circ}$, the polymer is too unflexible to "wrap" around the nanotube and the binding energy is significantly reduced (NOSC $=-58.45 \mathrm{kcal} \mathrm{mol}^{-1}$ ).

The higher affinity of NPSC for the carbonaceous surface can be attributed to the greater flexibility of the longer hydrophobic chains, which can 'wrap' around the nanotube, significantly increasing the van der Waals component of the interaction energy (Fig. 8). Increasing the number of polymer chains docked on the surface, from one to five, results in an increase in binding energy for all three polymer types. The overall effect of this is reduced for the NPSC because five chains are tightly packed around the surface of the nanotube (Fig. 9). However, the binding energy per chain for this polymer is still significantly higher than the other two, indicating that it is the most strongly bound of the three, in accordance

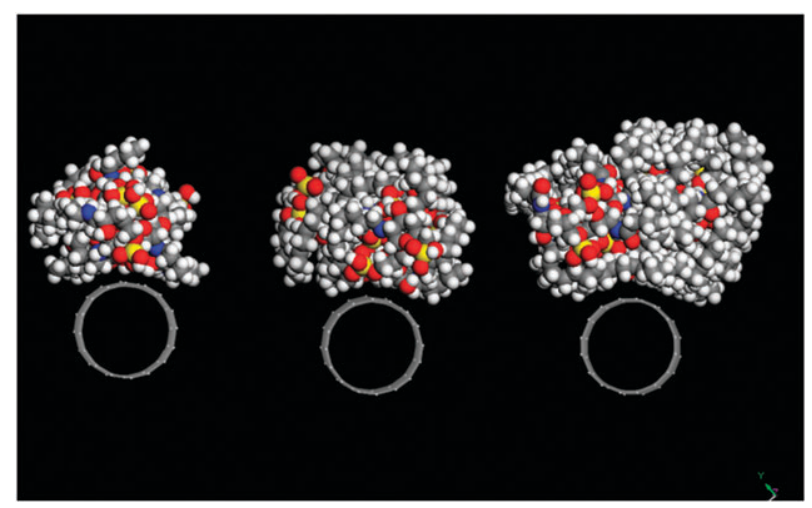

Fig. 8 The optimised location of a single polymer chain around a SWNT for NBSC, NOSC and NPSC, left to right respectively.

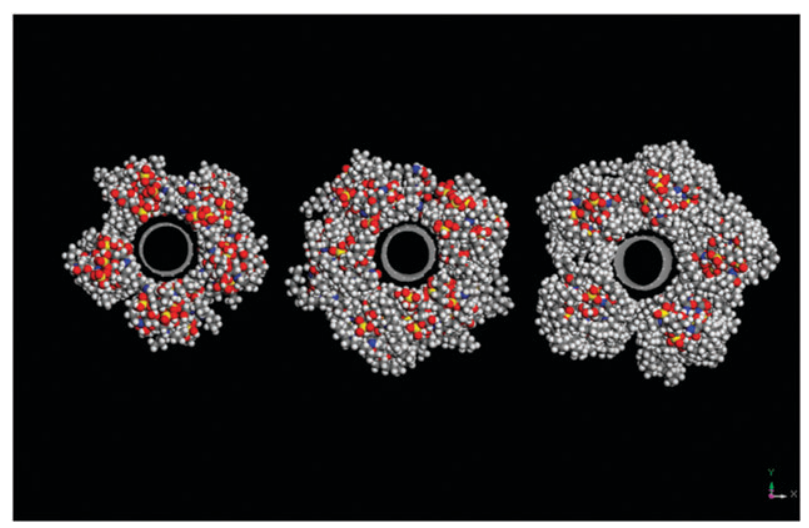

Fig. 9 The optimised location of five polymer chains around a SWNT for NBSC, NOSC and NPSC, left to right respectively. with the experimental measurements. Fig. 9 also shows that the thickness of the coating increases in the order NPSC $>$ NOSC $>$ NBSC in agreement with the observations from the AFM studies. The thickness of the polymer coating varies at different points along the tube, with average values estimated to be 3.9, 4.7 and $5.3 \mathrm{~nm}$ for NPSC, NOSC and NPSC, values that are in good general agreement with those observed from AFM (Table 2).

\section{Conclusions}

Both experimental and computational data agreed in indentifying NPSC as the best of the three polymers studied in wrapping around SWNTs and generating a stable aqueous dispersion of the carbonaceous nanostructure. Even though the polymer did not show anticoagulant activity as the NOSC derivative, the high value of cell viability after incubation with NPSC indicates that this is a good candidate for the preparation of biocompatible SWNT dipersions that could be used in biomedical and pharmaceutical applications.

\section{References}

1 A. Bianco, K. Kostarelos, C. D. Partidos and M. Prato, Chem. Commun., 2005, 571-577.

2 M. Foldvari and M. Bagonluri, Nanomed.: Nanotechnol., Biol. Med., 2008, 4, 173-182.

3 L. Lacerda, A. Bianco, M. Prato and K. Kostarelos, Adv. Drug Delivery Rev., 2006, 58, 1460-1470.

4 S. K. Smart, A. I. Cassady, G. Q. Lu and D. J. Martin, Carbon, 2006, 44, 1034-1047.

5 L. Lacerda, S. Raffa, M. Prato, A. Bianco and K. Kostarelos, Nano Today, 2007, 2, 38-43.

$6 \mathrm{~K}$. Kostarelos, L. Lacerda, G. Pastorin, W. Wu, WieckowskiSebastien, J. Luangsivilay, S. Godefroy, D. Pantarotto, J.-P. Briand, S. Muller, M. Prato and A. Bianco, Nat. Nanotechnol., 2007, 2, 108-113.

7 L. Panchakarla and A. Govindaraj, J. Chem. Sci., 2008, 120, 607-611.

8 Q. Zheng, Q. Xue, K. Yan, L. Hao, Q. Li and X. Gao, J. Phys. Chem. C, 2007, 111, 4628-4635.

9 J. Gou, B. Minaie, B. Wang, Z. Liang and C. Zhang, Computational Materials Science, 2004, 31, 225-236.

10 M. Yang, V. Koutsos and M. Zaiser, J. Phys. Chem. B, 2005, 109 10009-10014.

11 L. Zhang, T. Tao and C. Li, Polymer, 2009, 50, 3835-3840.

12 M. Roldo, K. Power, J. R. Smith, P. A. Cox, K. Papagelis, N. Bouropoulos and D. G. Fatouros, Nanoscale, 2009, 1, 366-396.

13 D. G. Fatouros, K. Power, O. Kadir, I. Dekany, S. N. Yannopoulos, N. Bouropoulos, A. Bakandritsos and M. Roldo, in preparation.

14 C. Y. Choi, S. B. Kim, P. K. Pak, D. I. Yoo and Y. S. Chung, Carbohydr. Polym., 2007, 68, 122-127.

15 L. Yuan, Z. Yue, H. Chen, H. Huang and T. Zhao, Colloids Surf., $B, 2009, \mathbf{7 3}, 346-350$.

16 C. Zhang, P. Qineng and H. Zhang, Colloids Surf., B, 2004, 39, 69-75.

17 A. Jos, S. Pichardo, M. Puerto, E. Sánchez, A. Grilo and A. M. Cameán, Toxicol. in Vitro, 2009, 23, 1491-1496.

18 E. Vázquez, V. Georgakilas and M. Prato, Chem. Commun., 2002, 2308-2309.

19 A. Z. F. Li, A. G. H. Luo, A. W. P. Zhou, W. A. F., A. R. Xiang and A. Y. P. Liu, Nanotechnology, 2006, 17, 3692-3698.

20 J. R. Maple, M. J. Hwang, T. P. Stockfish, U. Dinur, M. Waldman, C. S. Ewig and A. T. Hagler, J. Comput. Chem., 1994, 15, 162-182.

21 H. Li, J. Liu, S. Ding, C. Zhang, W. Shen and Q. You, Int. J. Biol. Macromol., 2009, 44, 249-256.

22 F. Li, W. G. Liu and K. D. Yao, Biomaterials, 2002, 23, 343-347. 
23 C. Le Tien, M. Lacroix, P. Ispas-Szabo and M.-A. Mateescu, J. Controlled Release, 2003, 93, 1-13.

24 Y. Wu, T. Seo, T. Sasaki, S. Irie and K. Sakurai, Carbohydr. Polym., 2006, 63, 493-499.

25 P. Vongchan, W. Sajomsang, W. Kasinrerk, D. Subyen and P. Kongtawelert, ScienceAsia, 2003, 29, 115-120.

26 R. Huang, Y. Du, J. Yang and L. Fan, Carbohydr. Res., 2003, 338, 483-489.

27 P. Vongchan, W. Sajomsang, D. Subyen and P. Kongtawelert, Carbohydr. Res., 2002, 337, 1239-1242.

28 R. Huang, Y. Du, L. Zheng, H. Liu and L. Fan, React. Funct. Polym., 2004, 59, 41-51.

29 G. Vikhoreva, G. Bannikova, P. Stolbushkina, A. Panov, N. Drozd, V. Makarov, V. Varlamov and L. Gal'braikh, Carbohydr. Polym., 2005, 62, 327-332.
30 R. Xing, H. Yu, S. Liu, W. Zhang, Q. Zhang, Z. Li and P. Li, Bioorg. Med. Chem., 2005, 13, 1387-1392.

31 Y. Zou and E. Khor, Carbohydr. Polym., 2009, 77, 516-525.

32 S. Green, M. Roldo, D. Douroumis, N. Bouropoulos, D. Lamprou and D. G. Fatouros, Carbohydr. Res., 2009, 344, 901-907.

33 G.-B. Jiang, D. Quan, K. Liao and H. Wang, Carbohydr. Polym., 2006, 66, 514-520.

34 M. J. O'Connell, P. Boul, L. M. Ericson, C. Huffman, Y. Wang, E. Haroz, C. Kuper, J. Tour, K. D. Ausman and R. E. Smalley, Chem. Phys. Lett., 2001, 342, 265-271.

35 V. Zorbas, A. Ortiz-Acevedo, A. B. Dalton, M. M. Yoshida, G. R. Dieckmann, R. K. Draper, R. H. Baughman, M. Jose-Yacaman and I. H. Musselman, J. Am. Chem. Soc., 2004, 126, 7222-7227.

36 E. F. Franca, R. D. Lins, L. C. G. Freitas and T. P. Straatsma, J. Chem. Theory Comput., 2008, 4, 2141-2149. 